\title{
Predictors of Distant Metastasis after Radical Surgery Followed by Postoperative Radiotherapy with or without Chemotherapy for Oropharyngeal Cancer
}

\author{
Mi Joo Chung, MD \\ Yeon Sil Kim, MD² \\ Ji Yoon Kim, MD2 \\ Yun Hee Lee, MD2 \\ Ji Hyun Jang, MD2 \\ Jin Hyoung Kang, $\mathrm{MD}^{3}$ \\ le Ryung Yoo, MD ${ }^{4}$ \\ Youn Soo Lee, MD
}

${ }^{1}$ Department of Radiation Oncology, Kyung Hee University Hospital at Gangdong, Seoul, Departments of ${ }^{2}$ Radiation Oncology,

${ }^{3}$ Medical Oncology, ${ }^{4}$ Nuclear Medicine, and ${ }^{5}$ Hospital Pathology,

Seoul St. Mary's Hospital,

College of Medicine,

The Catholic University of Korea, Seoul, Korea

Correspondence: Yeon Sil Kim, MD

Department of Radiation Oncology,

Seoul St. Mary's Hospital, College of Medicine,

The Catholic University of Korea,

222 Banpo-daero, Seocho-gu, Seoul 06591, Korea

Tel: 82-2-2258-6259

Fax: 82-2-2258-1532

E-mail: yeonkim7@catholic.ac.kr

Received October 7, 2015

Accepted February 11, 2016

Published Online March 3, 2016

\section{Purpose}

We investigated the prognostic factors for distant metastasis (DM) in patients with locally advanced oropharyngeal cancer (OPC) treated with surgery and adjuvant radiotherapy with or without concurrent chemotherapy.

\section{Materials and Methods}

Eighty-five patients treated between January 1995 and August 2014 were evaluated retrospectively. Data regarding the pathological tumour and nodal status, human papillomavirus (HPV) status, treatment characteristics, and pretreatment maximum standardized uptake value (SUVmax) of 18-fluoro-2-deoxyglucose positron emission tomography-computed tomography scan ( ${ }^{18} \mathrm{~F}-\mathrm{FDG}$ PET-CT) were evaluated, and their influence on DM and survival outcomes were analyzed.

\section{Results}

Median follow-up period was 48.0 months. Recurrence was observed in 20 patients, including locoregional recurrence and DM. DM was observed in 13 patients. A multivariate analysis confirmed that the presence of lymphovascular invasion $(p=0.031)$, lower neck lymph node $(L N)$ involvement $(p=0.006)$, SUVmax $\geq 9.7 \quad(p=0.014)$, and tumour size $\geq 3 \mathrm{~cm}$ ( $p=0.037$ ) significantly affected DM. HPV status was not associated with DM. Perineural invasion ( $p=0.048$ ), lower neck LN involvement ( $p=0.008)$, SUVmax $\geq 9.7(p=0.019)$, and tumour size $\geq 3 \mathrm{~cm}$ ( $p=0.033$ ) were also significant factors for the DM-free survival rate.

\section{Conclusion}

Lower neck LN involvement, high SUVmax in pretreatment ${ }^{18} \mathrm{~F}-\mathrm{FDG}$ PET-CT, and large tumour size were predictive factors for DM in patients of OPC.

\section{Introduction}

The incidence of oropharyngeal cancer (OPC) has increased over the past few years in Western countries as well as in Asia including Korea [1].

Numerous trials have recommended primary resection followed by radiotherapy (RT) with or without concurrent chemotherapy for locally advanced OPC with high-risk features, including extra-capsular nodal extension (ECE), posi-

\section{Key words}

Oropharyngeal neoplasms, Distant metastasis, Lower neck involvement, Radiotherapy, SUVmax, Pretreatment ${ }^{18}$ F-FDG PET-CT scan 
the DM rate. The chance of cure is very low when DM occurs, and OS decreases dramatically [4]. Although several studies have focused on LR control for head and neck cancers, few of these studies determined the prognostic factors for DM in patients with OPC treated with primary resection followed by RT with or without concurrent chemotherapy $[5,6]$. We could identify strategies that would reduce DM risk using accurate tools to predict the risk. Use of appropriate strategies can lead to decreased rates of distant failure and ultimately help improve OS for patients with high-risk OPC after adjuvant RT with or without concurrent chemotherapy. Thus, the aim of this study was to investigate prognostic factors for DM in patients who undergo primary resection with adjuvant RT with or without concurrent chemotherapy for locally advanced OPC.

\section{Materials and Methods}

\section{Patients}

We reviewed the medical records of patients with locally advanced OPC treated with curative resection followed by RT with or without concurrent chemotherapy between January 1995 and August 2014. The inclusion criteria for this study were (1) histologically proven squamous cell carcinoma of OPC; (2) Eastern Cooperative Oncology Group (ECOG) performance status $0-2$; (3) pT3 or pT4a and any nodal stage; (4) any T stage and N2-3; and (5) pT2 and N0 or N1 with unfavourable pathological findings, including ECE, positive RM, PNI, or lymphovascular invasion (LVI). Patients with DM of OPC at diagnosis were excluded. Finally, 85 patients were eligible. The TNM stages of the patients were re-classified according to the seventh edition of the American Joint Committee on Cancer staging system. All medical records were reviewed, including radiological images, pathology, including immunohistochemistry of $\mathrm{Ki}-67, \mathrm{p} 53$, and human papillomavirus (HPV) status, surgery, chemotherapy, and RT. Institutional review board approval was obtained prior to the study.

\section{Treatment}

\section{1) Surgery}

The pretreatment evaluation included history and physical examination; renal, hepatic, and bone marrow function tests; computed tomography (CT) or magnetic resonance imaging (MRI) of the head and neck and chest imaging, as clinically indicated. All patients underwent surgery with curative intent by two highly specialised head and neck surgeons. Neck dissections were performed in 85 patients at the time of surgery. Bilateral modified radical neck node dissection (MRND) was performed in 12 patients, ipsilateral MRND and contralateral selective neck dissection (SND) in 52 patients, ipsilateral MRND in 11 patients, ipsilateral SND in five patients, and bilateral SND in five patients.

\section{2) Radiation therapy}

Patients received two-dimensional RT between 1995 and 2000 and three-dimensional conformal RT or intensity-modulated RT (IMRT) was performed after 2000. A total dose of 54-66 Gy with conventional fractionation (1.8-2.12 Gy daily) was planned for all patients. The clinical target volumes (CTV) were classified according to three groups; high-risk CTV was defined as the primary tumour bed and pathologically positive lymph node (LN) stations, intermediate-risk CTV was defined as ipsilateral neck LNs and occasionally the contralateral level II LN area, and low-risk CTV was defined as the contralateral pathologically negative LN levels. CTV was expanded to consider setup uncertainty, and the appropriate planning target volume was designed with an additional 0.3-0.5 cm from CTV. Cases of involved RM and ECE were boosted up to $66 \mathrm{~Gy}$. All patients who underwent IMRT were treated after daily verification with volumetric image-guided RT.

\section{3) Chemotherapy}

Before making a decision of postoperative adjuvant treatment modality, the patient's age, performance status, co-morbidity, and pathology were evaluated by our multidisciplinary team. Adjuvant concurrent CRT was recommended to the patients of involved RM or ECE of LN. However, adjuvant concurrent CRT was considered in patients with other multiple risk factors including advanced T stage, multiple LN involvements, PNI, or LVI. Adjuvant therapy to decide RT or CRT was discussed considering high risk pathological features, patient's performance status, and co-morbidity together. In spite of involved RM or ECE, chemotherapy could not be given to old or co-morbid patients.

The chemotherapy regimen consisted of weekly doses of $30 \mathrm{mg}$ cisplatin $/ \mathrm{m}^{2}$ body surface area (BSA) and $400 \mathrm{mg}$ 5 -fluorouracil $/ \mathrm{m}^{2}$ BSA by continuous intravenous infusion for 1 week in 43 of the 52 patients who received CRT. Besides these regimens, docetaxel, cisplatin, and 5-fluorouracil were administered to seven patients, and two patients received cetuximab and cisplatin. Chemotherapy was withheld in patients who developed grade 3 or higher neutropenia, thrombocytopenia, liver, or renal toxicity. 


\section{Follow-up}

Patients were evaluated weekly by physical examination and appropriate blood tests during treatment. The patients were followed by all members of a multidisciplinary team at 1- to 3-month intervals for the first 2 years, and then every 6 months thereafter until 5 years after surgery. Thorough physical examination and imaging studies (neck CT and/or neck MRI, 18-fluoro-2-deoxyglucose positron emission tomography-computed tomography [PET-CT] and chest CT scans) were performed at each follow-up visit.

\section{Statistical analysis}

Distant metastasis-free survival (DMFS) was defined as time from operation date to DM, which ever occurred first. OS was defined as time from operation date to any cause of death or end of follow-up. DMFS and OS rates were estimated by using the Kaplan-Meier method. The log-rank test and Cox proportional hazards model were applied for identification of prognostic factors independently associated with $\mathrm{DM}$ and to estimate the hazard ratio (HR). Factors with p-values of $<0.25$ in a univariate analysis were included in a multivariate analysis. Two-sided $p$-values of $<0.05$ were considered significant. Correlation with HPV status and Ki-67, smoking, and p53 mutation was also analyzed using the chisquare test. All analyses were performed using SPSS ver. 12.0 (SPSS Inc., Chicago, IL).

\section{Results}

\section{Patient characteristics}

The patient characteristics are described in Table 1. The study cohort consisted of 81 men and four women with a median age of 58 years (range, 31 to 88 years). Of the 85 patients, 46 were current smokers, of whom 38 had a smoking history of $\geq 10$ pack-years. Eighty-four patients (98.8\%) had an ECOG performance status of $0-1$. The primary tumour sites were the tonsils in 63 patients $(74.1 \%)$, the base of the tongue in $19(22.4 \%)$, and the soft palate in three $(3.5 \%)$. Tumour stages were pT1-2 in 67 patients $(78.8 \%)$ and pT3-4 in 18 (21.2\%). Pathological LN stages were N0-2a in 32 patients $(37.6 \%)$ and N2b-3 in $53(62.4 \%)$. Seventy-five patients $(88.3 \%)$ had well and moderately differentiated tumours.

LVI and ECE were observed in $52(61.2 \%)$ and $38(44.7 \%)$ patients, respectively. Surgical RM involvement was observed in 37 of the 85 patients (43.5\%). The presence of PNI was observed in six patients (7.1\%). HPV positivity was observed in 37 patients (43.5\%). HPV-positive tumours were defined as specific in situ hybridization staining of tumour cell nuclei for HPV or positive p16 expression in an immunochemical analysis before 2013 and detection of HPV DNA by polymerase chain reaction after 2013. Lower neck LN (level IV and VB) involvement was observed in 17 patients (20.0\%). Ki-67 index $>50 \%$ was observed in 32 patients $(37.6 \%)$, p53 mutation was observed in 21 patients $(24.7 \%)$. The median pretreatment maximum standardized uptake value (SUVmax) of primary tumours was 9.7, and a SUVmax $\geq 9.7$ was observed in 30 patients $(35.3 \%)$. Tumour size $\geq 3 \mathrm{~cm}$ was observed in 48 patients (56.5\%).

\section{Prognostic factors affecting DM}

After a median follow-up period of 48.0 months (range, 5.3 to 189.2 months), recurrence was observed in 20 patients, including LR recurrence and DM. LR recurrence was observed in seven patients (8.2\%). DM was observed in 13 patients $(15.3 \%$; lungs in eight patients, liver and bone in four, and peritoneal seeding in one). Most DM (76.9\% of patients) occurred within 1 year after treatment, and the median time of DM was 9.43 months (range, 2.5 to 51.0 months). The result of univariate analysis for factors associated with DM is shown in Table 2. Three factors showed correlation with DM in the univariate analysis, including lower neck LN involvement, SUVmax $\geq 9.7$, and tumour size $\geq 3 \mathrm{~cm}$. No significant association was found between any other factor and DM. The multivariate logistic regression analysis showed that all the three factors in univariate analysis, lower neck LN involvement (HR, 77.394; 95\% confidence interval [CI], 3.506 to 1,708.536; $\mathrm{p}=0.006$ ), SUVmax $\geq 9.7$ (HR, $57.713 ; 95 \% \mathrm{CI}, 2.24$ to $1,484.920 ; \mathrm{p}=0.014)$, tumour size $\geq 3 \mathrm{~cm}$ (HR, 41.604; 95\% CI, 1.261 to 1,372.724; $\mathrm{p}=0.037$ ), and the presence of LVI (HR, 26.441; 95\% CI, 1.339 to 522.179; $\mathrm{p}=0.031$ ) were significant adverse factors affecting DM (Table 3). HPV status was not associated with DM.

\section{Survival outcomes}

The 5-year DMFS and OS rates are summarized in Table 4. The presence of PNI ( $\mathrm{p}=0.048)$, lower neck LN involvement ( $\mathrm{p}=0.008), \mathrm{SUVmax} \geq 9.7(\mathrm{p}=0.019)$, and tumour size more than $3 \mathrm{~cm}(\mathrm{p}=0.033)$ were significant prognostic factors for DMFS (Fig. 1). The presence of PNI ( $p=0.001)$ and lower neck LN involvement $(\mathrm{p}=0.028)$ were significant factors for OS.

\section{Salvage therapy}

Three of the seven patients with LR recurrence underwent 
Table 1. Patients' characteristics

\begin{tabular}{|c|c|}
\hline Characteristic & No. $(\%)(n=85)$ \\
\hline \multicolumn{2}{|l|}{ Age (yr) } \\
\hline$\leq 58$ & $43(50.6)$ \\
\hline$>58$ & $42(49.4)$ \\
\hline \multicolumn{2}{|l|}{ Sex } \\
\hline Male & $81(95.3)$ \\
\hline Female & $4(4.7)$ \\
\hline \multicolumn{2}{|l|}{ Smoking } \\
\hline Non-smoker & $39(45.9)$ \\
\hline Current smoker & $46(54.1)$ \\
\hline \multicolumn{2}{|c|}{ Smoking dose (pack-years) } \\
\hline$<10$, light & $8(9.4)$ \\
\hline$\geq 10$, heavy & $38(44.7)$ \\
\hline \multicolumn{2}{|l|}{ ECOG PS } \\
\hline 0 & $69(81.2)$ \\
\hline 1 & $15(17.6)$ \\
\hline 2 & $1(1.2)$ \\
\hline \multicolumn{2}{|l|}{ Disease site } \\
\hline Tonsil & $63(74.1)$ \\
\hline Base of tongue & $19(22.4)$ \\
\hline Soft palate & $3(3.5)$ \\
\hline \multicolumn{2}{|l|}{ Pathologic T stage } \\
\hline $\mathrm{T} 1$ & $25(29.4)$ \\
\hline $\mathrm{T} 2$ & $42(49.4)$ \\
\hline T3 & $14(16.5)$ \\
\hline $\mathrm{T} 4$ & $4(4.7)$ \\
\hline \multicolumn{2}{|l|}{ Pathologic N stage } \\
\hline N0 & $11(12.9)$ \\
\hline N1 & $13(15.3)$ \\
\hline $\mathrm{N} 2 \mathrm{a}$ & $8(9.4)$ \\
\hline $\mathrm{N} 2 \mathrm{~b}$ & $45(52.9)$ \\
\hline $\mathrm{N} 2 \mathrm{c}$ & $6(7.1)$ \\
\hline N3 & $2(2.4)$ \\
\hline \multicolumn{2}{|l|}{ Tumor size $(\mathrm{cm})$} \\
\hline$<3$ & $37(43.5)$ \\
\hline$\geq 3$ & $48(56.5)$ \\
\hline \multicolumn{2}{|l|}{ Tumor differentiation } \\
\hline Well and moderate & $75(88.3)$ \\
\hline Poor & $10(11.8)$ \\
\hline \multicolumn{2}{|c|}{ Lymphovascular invasion } \\
\hline No & $33(38.8)$ \\
\hline Yes & $52(61.2)$ \\
\hline \multicolumn{2}{|l|}{ Extracapsular spread } \\
\hline No & $47(55.3)$ \\
\hline Yes & $38(44.7)$ \\
\hline \multicolumn{2}{|c|}{ Surgical margin involvement } \\
\hline No & $48(56.5)$ \\
\hline Yes & $37(43.5)$ \\
\hline \multicolumn{2}{|c|}{ Perineural involvement } \\
\hline No & $79(92.9)$ \\
\hline Yes & $6(7.1)$ \\
\hline
\end{tabular}

Table 1. Continued

$\begin{array}{lc}\text { Characteristic } & \text { No. }(\%)(\mathbf{n}=85) \\ \text { HPV status } & \\ \text { Negative } & 48(56.5) \\ \text { Positive } & 37(43.5) \\ \text { Lower neck involvement } & \\ \text { No } & 68(80.0) \\ \text { Yes } & 17(20.0) \\ \text { SUVmax } & \\ <9.7 & 37(43.5) \\ \geq 9.7 & 30(35.3) \\ \text { Unknown } & 18(21.2) \\ \text { Adjuvant therapy } & \\ \text { CCRT } & 52(61.2) \\ \text { RT alone } & 33(38.8)\end{array}$

ECOG PS, Eastern Cooperative Oncology Group performance status; HPV, human papillomavirus; SUVmax, maximum standardized uptake value; CCRT, concurrent chemoradiotherapy.

salvage RT. One patient received chemotherapy, one underwent salvage surgery, and the remaining patient refused additional therapy. Four of the 13 patients with DM underwent metastasectomy of the lungs or liver. Six patients underwent systemic chemotherapy, and one patient underwent palliative RT. Two patients did not undergo additional therapy because of relatively poor tolerance. Correlation with HPV status and Ki-67, smoking, and p53 mutation, HPV-positive tumour showed high Ki-67 staining. The median Ki-67 index was 50 and 23 of 37 HPV-positive tumours $(62.2 \%)$ showed a Ki-67 index $>50 \%$ compared with that of HPV-negative tumours $(p<0.001)$ (Table 5$)$. The HPV-positive group showed a $27.0 \%$ rate of p53 mutations, and $48.6 \%$ of the patients having a heavy smoking history. No difference was detected between HPV status and p53 mutation and smoking history.

\section{Characteristics according to adjuvant therapy}

Results of univariate analysis according to adjuvant therapy are shown in Table 6 . Age $(\mathrm{p}=0.015)$, pathologic $\mathrm{N}$ stage $(p=0.042)$, the presence of ECE $(p<0.001)$, and number of positive LN $(\mathrm{p}=0.023)$ were significantly different between the postoperative CRT group and the postoperative RT group. 
Table 2. Univariate analysis of factors associated with distant metastasis

\begin{tabular}{|c|c|c|}
\hline Characteristic & Unadjusted hazard ratio (95\% CI) & p-value \\
\hline \multicolumn{3}{|l|}{ Pathologic T stage } \\
\hline T1-2 & 1 & \\
\hline T3-4 & $1.852(0.569-6.027)$ & 0.306 \\
\hline \multicolumn{3}{|l|}{ Pathologic N stage } \\
\hline N0-2a & 1 & \\
\hline $\mathrm{N} 2 \mathrm{~b}-3$ & $3.929(0.811-19.031)$ & 0.089 \\
\hline \multicolumn{3}{|l|}{ Tumor differentiation } \\
\hline Well and moderate & 1 & \\
\hline Poor & $1.455(0.272-7.776)$ & 0.661 \\
\hline \multicolumn{3}{|c|}{ Lymphovascular invasion } \\
\hline No & 1 & \\
\hline Yes & $4.291(0.926-19.891)$ & 0.063 \\
\hline \multicolumn{3}{|l|}{ Extracapsular spread } \\
\hline No & 1 & \\
\hline Yes & $1.860(0.608-5.691)$ & 0.277 \\
\hline \multicolumn{3}{|c|}{ Surgical margin involvement } \\
\hline No & 1 & \\
\hline Yes & $1.013(0.310-3.313)$ & 0.983 \\
\hline \multicolumn{3}{|l|}{ Perineural involvement } \\
\hline No & 1 & \\
\hline Yes & $4.219(0.926-19.891)$ & 0.063 \\
\hline \multicolumn{3}{|l|}{ HPV status } \\
\hline Negative & 1 & \\
\hline Positive & $1.134(0.346-3.712)$ & 0.836 \\
\hline \multicolumn{3}{|c|}{ Lower neck involvement } \\
\hline No & 1 & \\
\hline Yes & $3.739(1.256-11.130)$ & 0.018 \\
\hline \multicolumn{3}{|l|}{ Tumor size $(\mathrm{cm})$} \\
\hline$<3$ & 1 & \\
\hline$\geq 3$ & $5.500(1.138-26.592)$ & 0.034 \\
\hline \multicolumn{3}{|c|}{ No. of positive lymph nodes } \\
\hline$<4$ & 1 & \\
\hline$\geq 4$ & $2.773(0.820-9.379)$ & 0.101 \\
\hline \multicolumn{3}{|l|}{ Depth of invasion $(\mathrm{cm})$} \\
\hline$<1.23$ & 1 & \\
\hline$\geq 1.23$ & $1.556(0.469-5.160)$ & 0.470 \\
\hline \multicolumn{3}{|l|}{ SUVmax } \\
\hline$<9.7$ & 1 & \\
\hline$\geq 9.7$ & $8.554(1.027-71.226)$ & 0.047 \\
\hline \multicolumn{3}{|l|}{ Age (yr) } \\
\hline$\leq 60$ & 1 & \\
\hline$>60$ & $0.397(0.101-1.564)$ & 0.187 \\
\hline \multicolumn{3}{|l|}{ Adjuvant therapy } \\
\hline CCRT & 1 & \\
\hline RT & $0.982(0.292-3.306)$ & 0.977 \\
\hline \multicolumn{3}{|l|}{ RT duration (wk) } \\
\hline$<9$ & 1 & \\
\hline$\geq 9$ & $0.917(0.101-8.311)$ & 0.938 \\
\hline \multicolumn{3}{|c|}{ Duration between surgery and RT (wk) } \\
\hline$<6$ & 1 & \\
\hline$\geq 6$ & $0.659(0.185-2.343)$ & 0.519 \\
\hline
\end{tabular}


Table 2. Continued

\begin{tabular}{lcc} 
Characteristic & Unadjusted hazard ratio $(95 \%$ CI) & p-value \\
No. of chemotherapy cycles & 1 & \\
$\quad 5-7$ & $2.250(0.243-20.837)$ & 0.475 \\
$\quad<5$ & 1 & \\
Smoking dose (pack-years) & $3.724(0.923-15.029)$ & 0.065 \\
$\quad<10$, light & & \\
$\quad \geq 10$, heavy & & \\
\hline
\end{tabular}

CI, confidence interval; HPV, human papillomavirus; SUVmax, maximum standardized uptake value; CCRT, concurrent chemoradiotherapy; RT, radiotherapy.

Table 3. Multivariate analysis of factors associated with distant metastasis

\begin{tabular}{|c|c|c|}
\hline Characteristic & Hazard ratio $(95 \% \mathrm{CI})$ & p-value \\
\hline \multicolumn{3}{|c|}{ Pathologic N stage } \\
\hline N0-2a & 1 & \\
\hline $\mathrm{N} 2 \mathrm{~b}-3$ & $1.641(0.113-23.748)$ & 0.717 \\
\hline \multicolumn{3}{|c|}{ Lymphovascular invasion } \\
\hline No & 1 & \\
\hline Yes & 26.441 (1.339-522.179) & 0.031 \\
\hline \multicolumn{3}{|c|}{ Perineural involvement } \\
\hline No & 1 & \\
\hline Yes & $1.662(0.020-140.889)$ & 0.822 \\
\hline \multicolumn{3}{|c|}{ Lower neck involvement } \\
\hline No & 1 & \\
\hline Yes & $77.394(3.506-1,708.536)$ & 0.006 \\
\hline \multicolumn{3}{|l|}{ SUVmax } \\
\hline$<9.7$ & 1 & \\
\hline$\geq 9.7$ & $57.713(2.243-1,484.920)$ & 0.014 \\
\hline \multicolumn{3}{|l|}{ Tumor size $(\mathrm{cm})$} \\
\hline$<3$ & 1 & \\
\hline$\geq 3$ & $41.604(1.261-1,372.724)$ & 0.037 \\
\hline
\end{tabular}

CI, confidence interval; SUVmax, maximum standardized uptake value.

\section{Discussion}

For high-risk patients with locally advanced OPC, including those with positive RM, the presence of ECE, and multiple LN metastasis, LR recurrence, and DM were common after primary surgery [2]. Therefore, postoperative CRT is necessary in these patients, with the expectation that it will provide improved LR control and a better OS rate. Previous studies have shown that postoperative CRT for locally advanced squamous cell carcinoma of the head and neck change LR control and OS rates. The RTOG 9501 and the EORTC 22931 trials demonstrated that postoperative CRT is a more effective treatment in terms of LR control and OS rate than that of adjuvant RT alone [2]. However, neither study showed any effect on distant control. Therefore, postoperative CRT does not reduce the probability of DM, despite use of high-dose cisplatin.

We analyzed only OPC patients who underwent surgery and adjuvant therapy in our hospital. And as possible prognostic factors for DM, we found that lower neck involvement, high SUVmax on pretreatment PET-CT, and large tumour size $\geq 3 \mathrm{~cm}$ were significant prognostic factors for $\mathrm{DM}$. These findings suggest that further adjuvant chemotherapy or more effective novel chemotherapy regimens are needed to reduce DM in patients with high-risk OPC. Judicious use of adjuvant therapy could not only reduce the DM rate but also improve the OS rate in high-risk patients with 
Table 4. Five-year Kaplan-Meier DMFS and OS according to the prognostic factors

\begin{tabular}{|c|c|c|c|c|c|}
\hline Characteristic & No. of patients & 5-Yr DMFS (\%) & p-value & 5-Yr OS (\%) & p-value \\
\hline \multicolumn{6}{|c|}{ Pathologic N stage } \\
\hline N0-2a & 32 & 93.7 & 0.078 & 68.4 & 0.796 \\
\hline $\mathrm{N} 2 \mathrm{~b}-3$ & 53 & 74.0 & & 68.7 & \\
\hline \multicolumn{6}{|c|}{ Lymphovascular invasion } \\
\hline No & 33 & 93.8 & 0.121 & 68.0 & 0.812 \\
\hline Yes & 52 & 75.5 & & 68.9 & \\
\hline \multicolumn{6}{|c|}{ Perineural involvement } \\
\hline No & 79 & 83.0 & 0.048 & 73.0 & 0.001 \\
\hline Yes & 6 & None & & 0.0 & \\
\hline \multicolumn{6}{|c|}{ Lower neck involvement } \\
\hline No & 68 & 88.3 & 0.008 & 70.3 & 0.028 \\
\hline Yes & 17 & 54.1 & & 60.3 & \\
\hline \multicolumn{6}{|l|}{ SUVmax } \\
\hline$<9.7$ & 37 & 96.4 & 0.019 & 71.6 & 0.967 \\
\hline$\geq 9.7$ & 30 & 78.8 & & 71.5 & \\
\hline \multicolumn{6}{|l|}{ Tumor size $(\mathrm{cm})$} \\
\hline$<3$ & 37 & 94.0 & 0.033 & 75.5 & 0.562 \\
\hline$\geq 3$ & 48 & 72.7 & & 63.7 & \\
\hline
\end{tabular}

DMFS, distant metastasis-free survival; OS, overall survival; SUVmax, maximum standardized uptake value.

OPC.

In current $\mathrm{T}$ staging for OPC cancer, tumour size more than $2 \mathrm{~cm}$ and less than $4 \mathrm{~cm}$ is the same as T2. However, according to our result, tumour size more than $3 \mathrm{~cm}$ should be considered as a high-risk feature for DM. Likewise, in current $\mathrm{N}$ staging for $\mathrm{OPC}$, LN size or ipsilateral/bilateral involvement is considered to decide $\mathrm{N}$ staging. However, according to our result, the level of involvement should be considered together to decide more aggressive adjuvant therapy.

Ono et al. [7] reported on the unfavourable effect of lower neck LN involvement in patients with head and neck carcinoma. In their study of 338 patients who underwent neck dissection for head and neck carcinoma, an extremely poor survival rate was clearly demonstrated when LN metastases were confined to level IV. Our results also demonstrate that lower neck LN involvement was significantly associated with a high incidence of DM and a poor OS rate. Kim et al. [8], who analyzed prognostic factors for DM after induction chemotherapy followed by CRT for head and neck cancer, reported that the 5-year DMFS rates according to lower neck LN involvement (positive vs. negative) were $34.3 \%$ versus $55.2 \%$. Despite addition of induction chemotherapy prior to CRT to overcome DM, patients with lower neck LN involvement still showed a higher incidence of DM. Therefore, lower neck LN involvement might be regarded as a major factor when considering aggressive therapy.

Several studies have demonstrated that pretreatment
SUVmax is a good predictor of OS and disease-free survival (DFS) in patients with head and neck cancer [9-11]. Xie et al. [10] conducted a meta-analysis of survival data to determine the prognostic value of pretreatment SUV for OS. In their study, the risk of death decreased by $76 \%$ in patients with a low SUV. In a univariate analysis, Suzuki et al. [9] found that patients with hypopharyngeal squamous cell carcinoma and pretreatment SUVmax $\geq 13$ showed significantly shorter OS. We selected a SUVmax cut-off value of 9.7, which was the median pre-treatment SUVmax value. Although, SUVmax $\geq 9.7$ was an independent predictor of DM, SUVmax $\geq 9.7$ was not correlated with OS in our study. After 2004, 67 of 85 patients underwent PET-CT. We assumed that these small numbers prevented a statistically significant OS result.

Ang et al. [12] and the authors of several retrospective studies reported that patients with HPV-positive OPC cancer showed increased OS [13]. However, our results showed that HPV positivity was not significantly associated with higher OS or DFS, and the reason for this difference from previous studies is unclear.

In our study, 23 of $37 \mathrm{HPV}$-positive tumours (62.2\%) showed a Ki-67 index $>50 \%$ compared with that of HPVnegative tumours $(\mathrm{p}<0.001)$ (Table 5). A higher Ki-67 index in HPV-positive tumours was considered a confounding factor in our study.

Smoking and HPV are known major risk factors for OPC. Heavy smoking increases the frequency of p53 mutations, and the frequency of p53 mutations in smokers is twice as 

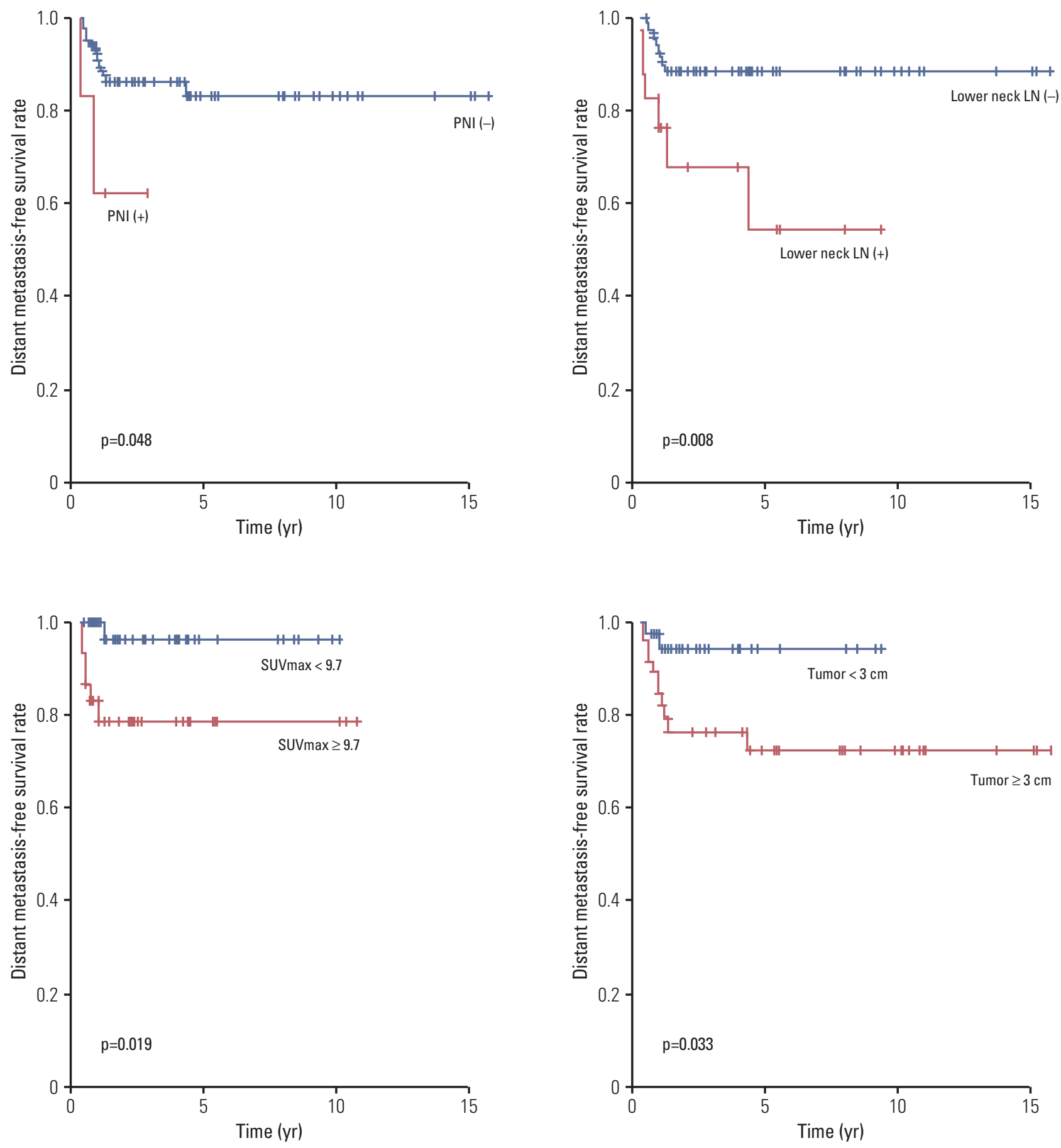

Fig. 1. Kaplan-Meier distant metastasis-free survival curve according to the prognostic factors. PNI, perineural invasion; LN, lymph node; SUVmax, maximum standardized uptake value.

high as that in non-smokers [14]. Previous studies have shown that patients with HPV-positive OPC are less likely to harbour p53 mutations compared to those with HPV-negative OPC [15]. Contrary to our expectation, the HPV-posi- tive group showed a $27.0 \%$ rate of p53 mutations, and $48.6 \%$ of the patients having a heavy smoking history (Table 5). No difference was detected between HPV status and p53 and smoking. We tentatively assumed that the combination of 
Table 5. Correlation with HPV status and Ki-67, smoking, and p53 mutation

\begin{tabular}{|c|c|c|c|}
\hline \multirow{2}{*}{ Characteristic } & \multicolumn{2}{|c|}{ HPV status } & \multirow{2}{*}{ p-value } \\
\hline & No & Yes & \\
\hline \multicolumn{4}{|l|}{ Ki-67 (\%) } \\
\hline$\leq 50$ & $39(81.3)$ & $14(37.8)$ & $<0.001$ \\
\hline$>50$ & $9(18.8)$ & $23(62.2)$ & \\
\hline \multicolumn{4}{|l|}{ Smoking } \\
\hline No & $28(58.3)$ & $19(51.4)$ & 0.340 \\
\hline Yes & $20(41.7)$ & $18(48.6)$ & \\
\hline \multicolumn{4}{|l|}{ p53 mutation } \\
\hline No & 37 (77.1) & $27(73.0)$ & 0.801 \\
\hline Yes & $11(22.9)$ & $10(27.0)$ & \\
\hline
\end{tabular}

Values are presented as number (\%). HPV, human papillomavirus.

Table 6. Characteristics according to adjuvant therapy

\begin{tabular}{|c|c|c|c|}
\hline \multirow{2}{*}{ Characteristic } & \multicolumn{2}{|c|}{ Adjuvant therapy } & \multirow{2}{*}{ p-value } \\
\hline & CCRT & RT alone & \\
\hline \multicolumn{4}{|l|}{ Age (yr) } \\
\hline$\leq 60$ & $32(61.5)$ & $11(33.3)$ & 0.015 \\
\hline$>60$ & $20(38.5)$ & $22(66.7)$ & \\
\hline \multicolumn{4}{|c|}{ Pathologic T stage } \\
\hline T1-2 & $43(82.7)$ & $24(72.7)$ & 0.290 \\
\hline T3-4 & $9(17.3)$ & $9(27.3)$ & \\
\hline \multicolumn{4}{|c|}{ Pathologic N stage } \\
\hline N0-2a & $15(28.8)$ & $17(51.5)$ & 0.042 \\
\hline $\mathrm{N} 2 \mathrm{~b}-3$ & $37(71.2)$ & $16(48.5)$ & \\
\hline \multicolumn{4}{|c|}{ Lymphovascular invasion } \\
\hline No & $19(36.5)$ & $13(39.4)$ & 0.821 \\
\hline Yes & $33(63.5)$ & $20(60.6)$ & \\
\hline \multicolumn{4}{|c|}{ Extracapsular spread } \\
\hline No & $20(38.5)$ & $27(81.8)$ & $<0.001$ \\
\hline Yes & $32(61.5)$ & $6(18.2)$ & \\
\hline \multicolumn{4}{|c|}{ Surgical margin involvement } \\
\hline No & $28(53.8)$ & $20(60.6)$ & 0.655 \\
\hline Yes & $24(46.2)$ & $13(39.4)$ & \\
\hline \multicolumn{4}{|c|}{ Perineural involvement } \\
\hline No & $49(94.2)$ & $30(90.9)$ & 0.673 \\
\hline Yes & $3(5.8)$ & $3(9.1)$ & \\
\hline \multicolumn{4}{|c|}{ No. of positive lymph nodes } \\
\hline$<4$ & $33(63.5)$ & $29(87.9)$ & 0.023 \\
\hline$\geq 4$ & $19(36.5)$ & $4(12.1)$ & \\
\hline
\end{tabular}

Values are presented as number (\%). CCRT, concurrent chemoradiotherapy; RT, radiotherapy.
HPV positivity and the intensity and duration of smoking played dual roles in the pathogenesis of OPC in the HPVpositive group. This assumption explains in part the lack of a significant difference in treatment outcomes between the HPV-positive and HPV-negative groups.

In the current study, we sought to identify factors to predict DM in patients with OPC. However, the study had some limitations. Sample size was limited to 85 patients. And the study was conducted retrospectively. The adjuvant treatment groups were divided into RT alone and CRT. Because we analyzed only resectable OPC, PNI (no $92.9 \%$ vs. yes $7.1 \%, \mathrm{p}=0.063$ ), lower neck $\mathrm{LN}$ involvement (no $80 \%$ vs. yes $20 \%, \mathrm{p}=0.018$ ) cases were relatively small in number and patient numbers with or without these risk factors were not well balanced. Despite these limitations, we identified meaningful prognostic factors regarding DM in OPC patients.

\section{Conclusion}

In conclusion, lower neck LN involvement, pretreatment SUVmax $\geq 9.7$, and tumour size $\geq 3 \mathrm{~cm}$ were predictors for $\mathrm{DM}$ in a multivariate analysis of patients with OPC who underwent radical surgery followed by adjuvant RT with or without chemotherapy. The 5-year OS rate in patients with PNI and lower neck LN involvement was low; thus, further investigation of adjuvant therapies in prospective studies is needed for patients who are at high risk for DM.

\section{Conflicts of Interest}

Conflict of interest relevant to this article was not reported. 


\section{References}

1. Shin A, Jung YS, Jung KW, Kim K, Ryu J, Won YJ. Trends of human papillomavirus-related head and neck cancers in Korea: national cancer registry data. Laryngoscope. 2013;123: E30-7.

2. Bernier J, Cooper JS, Pajak TF, van Glabbeke M, Bourhis J, Forastiere A, et al. Defining risk levels in locally advanced head and neck cancers: a comparative analysis of concurrent postoperative radiation plus chemotherapy trials of the EORTC (\#22931) and RTOG (\#9501). Head Neck. 2005;27: $843-50$

3. Shang J, Gu J, Han Q, Xu Y, Yu X, Wang K. Chemoradiotherapy is superior to radiotherapy alone after surgery in advanced squamous cell carcinoma of the head and neck: a systematic review and meta-analysis. Int J Clin Exp Med. 2014;7: 2478-87.

4. Cohen EE, Karrison TG, Kocherginsky M, Mueller J, Egan R, Huang $\mathrm{CH}$, et al. Phase III randomized trial of induction chemotherapy in patients with N2 or N3 locally advanced head and neck cancer. J Clin Oncol. 2014;32:2735-43.

5. Krishnatry R, Gupta T, Murthy V, Ghosh-Laskar S, Budrukkar A, Chaturvedi $\mathrm{P}$, et al. Factors predicting 'time to distant metastasis' in radically treated head and neck cancer. Indian J Cancer. 2014;51:231-5.

6. Li X, Di B, Shang Y, Zhou Y, Cheng J, He Z. Clinicopathologic risk factors for distant metastases from head and neck squamous cell carcinomas. Eur J Surg Oncol. 2009;35:1348-53.

7. Ono I, Ebihara S, Saito H, Yoshizumi T. Correlation between prognosis and degree of lymph node involvement in carcinoma of the head and neck. Auris Nasus Larynx. 1985;12 Suppl 2:S85-9.
8. Kim DH, Kim WT, Lee JH, Ki YK, Nam JH, Lee BJ, et al. Analysis of the prognostic factors for distant metastasis after induction chemotherapy followed by concurrent chemoradiotherapy for head and neck cancer. Cancer Res Treat. 2015;47: 46-54.

9. Suzuki H, Kato K, Fujimoto Y, Itoh Y, Hiramatsu M, Maruo T, et al. 18F-FDG-PET / CT predicts survival in hypopharyngeal squamous cell carcinoma. Ann Nucl Med. 2013;27:297-302.

10. Xie P, Li M, Zhao H, Sun X, Fu Z, Yu J. 18F-FDG PET or PETCT to evaluate prognosis for head and neck cancer: a metaanalysis. J Cancer Res Clin Oncol. 2011;137:1085-93.

11. Kim G, Kim YS, Han EJ, Yoo IR, Song JH, Lee SN, et al. FDG$\mathrm{PET} / \mathrm{CT}$ as prognostic factor and surveillance tool for postoperative radiation recurrence in locally advanced head and neck cancer. Radiat Oncol J. 2011;29:243-51.

12. Ang KK, Harris J, Wheeler R, Weber R, Rosenthal DI, NguyenTan PF, et al. Human papillomavirus and survival of patients with oropharyngeal cancer. N Engl J Med. 2010;363:24-35.

13. Joo YH, Cho KJ, Park JO, Nam IC, Kim CS, Kim MS. High-risk human papillomavirus and lymph node size in patients with single node metastasis of oral and oropharyngeal cancer. Acta Otolaryngol. 2014;134:395-400.

14. Ko Y, Abel J, Harth V, Brode P, Antony C, Donat S, et al. Association of CYP1B1 codon 432 mutant allele in head and neck squamous cell cancer is reflected by somatic mutations of p53 in tumor tissue. Cancer Res. 2001;61:4398-404.

15. Gillison ML, Koch WM, Capone RB, Spafford M, Westra WH, $\mathrm{Wu} \mathrm{L}$, et al. Evidence for a causal association between human papillomavirus and a subset of head and neck cancers. J Natl Cancer Inst. 2000;92:709-20. 